This article was downloaded by: [Max Planck Inst \& Research Groups Consortium]

On: 29 May 2009

Access details: Access Details: [subscription number 789998259]

Publisher Psychology Press

Informa Ltd Registered in England and Wales Registered Number: 1072954 Registered office: Mortimer House, 37-41 Mortimer Street, London W1T 3JH, UK

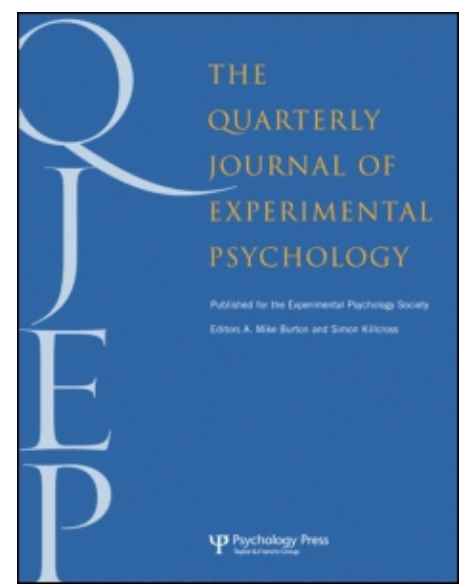

The Quarterly Journal of Experimental Psychology

Publication details, including instructions for authors and subscription information:

http://www.informaworld.com/smpp/title content=t716100704

\title{
Segmenting the body into parts: Evidence from biases in tactile perception
}

Frédérique de Vignemont a ; Asifa Majid ${ }^{\mathrm{b}}$; Corinne Jola ${ }^{\mathrm{c}}$; Patrick Haggard ${ }^{\mathrm{d}}$

${ }^{a}$ Institut Jean-Nicod, EHESS-ENS-CNRS, Paris, France ${ }^{\mathrm{b}}$ Max Planck Institute for Psycholinguistics,

Nijmegen, The Netherlands ${ }^{c}$ Department of Psychology and Social Sciences, City University, London, UK ${ }^{d}$

Institute of Cognitive Neuroscience and Department of Psychology, University College London, London, UK

First Published on: 03 May 2008

To cite this Article de Vignemont, Frédérique, Majid, Asifa, Jola, Corinne and Haggard, Patrick(2008)'Segmenting the body into parts: Evidence from biases in tactile perception', The Quarterly Journal of Experimental Psychology,62:3,500 — 512

To link to this Article: DOI: 10.1080/17470210802000802

URL: http://dx.doi.org/10.1080/17470210802000802

\section{PLEASE SCROLL DOWN FOR ARTICLE}

\footnotetext{
Full terms and conditions of use: http://www.informaworld.com/terms-and-conditions-of-access.pdf

This article may be used for research, teaching and private study purposes. Any substantial or systematic reproduction, re-distribution, re-selling, loan or sub-licensing, systematic supply or distribution in any form to anyone is expressly forbidden.

The publisher does not give any warranty express or implied or make any representation that the contents will be complete or accurate or up to date. The accuracy of any instructions, formulae and drug doses should be independently verified with primary sources. The publisher shall not be liable for any loss, actions, claims, proceedings, demand or costs or damages whatsoever or howsoever caused arising directly or indirectly in connection with or arising out of the use of this material.
} 


\title{
Segmenting the body into parts: Evidence from biases in tactile perception
}

\author{
Frédérique de Vignemont \\ Institut Jean-Nicod, EHESS-ENS-CNRS, Paris, France \\ Asifa Majid \\ Max Planck Institute for Psycholinguistics, Nijmegen, The Netherlands \\ Corinne Jola \\ Department of Psychology and Social Sciences, City University, London, UK \\ Patrick Haggard \\ Institute of Cognitive Neuroscience and Department of Psychology, University College London, London, UK
}

\begin{abstract}
How do we individuate body parts? Here, we investigated the effect of body segmentation between hand and arm in tactile and visual perception. In a first experiment, we showed that two tactile stimuli felt farther away when they were applied across the wrist than when they were applied within a single body part (palm or forearm), indicating a "category boundary effect". In the following experiments, we excluded two hypotheses, which attributed tactile segmentation to other, nontactile factors. In Experiment 2, we showed that the boundary effect does not arise from motor cues. The effect was reduced during a motor task involving flexion and extension movements of the wrist joint. Action brings body parts together into functional units, instead of pulling them apart. In Experiments 3 and 4, we showed that the effect does not arise from perceptual cues of visual discontinuities. We did not find any segmentation effect for the visual percept of the body in Experiment 3, nor for a neutral shape in Experiment 4. We suggest that the mental representation of the body is structured in categorical body parts delineated by joints, and that this categorical representation modulates tactile spatial perception.
\end{abstract}

Keywords: Body parts; Segmentation; Touch; Vision; Body schema.

The body is made up of parts. Body parts have hierarchical structure (e.g., the fingers are part of the hand) and topological relationships (e.g., the hand is contiguous to the arm). However, there

Correspondence should be addressed to Frédérique de Vignemont, Institut Jean Nicod, Département d'Etudes Cognitives, Ecole Normale Supérieure, 29 rue d’Ulm, 75005 Paris, France. E-mail: fvignemont@isc.cnrs.fr

This research was supported by a Marie Curie Fellowship and an Agence Nationale pour la Recherche (ANR) grant to F.V., a Biotechnology and Biological Sciences Research Council (BBSRC) grant to P.H., and a British Academy grant to P.H. and F.V. C.J. was supported by a grant from the Swiss National Foundation (106258). We thank Johan Weustink for technical support and Ludy Cillisen for assistance with Experiments 3 and 4. 
have been few systematic studies examining how the body is segmented into parts, and the representation of the boundaries of body parts has rarely been considered explicitly. Mereology (from the Greek $\mu \varepsilon \rho o \sigma$, "part") is the theory of parthood relations: of the relations of part to whole and the relations of part to part within a whole. The organizing principles of body mereology remain an open question in neuroscience, in neuropsychology, and also in linguistics (de Vignemont, Tsakiris, \& Haggard, 2005b).

Most studies of "structural body representation" take it for granted that mental body representation follows the terms of natural language (Schwoebel \& Coslett, 2005; Sirigu, Grafman, Bressler, \& Sunderland, 1991). Psychology and linguistics both recognize body parts as an important semantic category (Reed, McGoldrick, Shackelford, \& Fidopiastis, 2004). Psychological studies emphasize the visual features of body parts, individuating them by shape, size, and spatial orientation, or by "geons" delimited by visual discontinuities (Andersen, 1978; Biederman, 1987; Brown, 1976). Morrison and Tversky (2005), emphasized the "functional salience" of body parts, but did not explicitly define what this means. Body shape is relatively invariant across individuals within a species. Therefore body part categories should also be universal, at least if these are based on visual shape (Andersen, 1978). However, recent cross-cultural studies have shown that linguistic categorization of body parts can vary substantially (Majid, Enfield, \& van Staden, 2006). For instance, in some languages like the Papuan language of Rossel Island, there is just one term for hand and arm, referring to the whole upper limb (Levinson, 2006). This is not an exception to a general rule, as it is true in a wide number of languages (e.g., almost one third of the languages studied in Brown, 2005). The distinction between arm and hand, far from being obvious, thus raises questions about what determines parthood.

Knowledge of body parts can be specifically impaired at the linguistic level in body-specific aphasia (Laiacona, Allamano, Lorenzi, \& Capitani, 2006; Suzuki, Yamadori, \& Fujii, 1997), but also at the visuo-spatial level (Buxbaum \& Coslett, 2001) and at the motor level (Buxbaum, Giovannetti, \& Libon, 2000). Patients with autotopagnosia display a general impairment of the visuo-spatial organization of the body. They are impaired in judging distances between two body parts, in naming and identifying body parts that were touched, and in recognizing body parts from different visual perspectives. Similarly, patients with "numbsense" syndrome who only unconsciously perceive tactile stimulations are not able to verbally localize where they have been touched. However, they may be able to point towards the part of their body where they were touched (Rossetti, Rode, \& Boisson, 1995). Their visuo-spatial body map is impaired, but not their motor body map. We can contrast numbsense patients with deafferented patients, who receive no proprioceptive and tactile information, but only information about heat and cold. These latter patients are able to identify the body part that is stimulated, but not to point toward the stimulated body part (Paillard, 1999). Their motor body map is impaired, but not their visuo-spatial body map. Similarly, patients with apraxia cannot individuate relevant body parts to move in order to imitate meaningless gestures and to imagine movements in motor imagery tasks (Buxbaum et al., 2000; Goldenberg, 1995).

The dissociations between autotopagnosia and numbsense on the one hand and deafferentation and apraxia on the other hand argue in favour of more than one spatial representation of the organization of body parts (Gallagher \& Cole, 1995; Paillard, 1999; Schwoebel \& Coslett, 2005; Sirigu et al., 1991). The way we use information determines the way we encode it (Milner \& Goodale, 1995). Like vision and audition, somatosensory processing can be either recognition oriented or action oriented (Dijkerman \& de Haan, 2007). Thus, primary somatosensory cortex (SI) may mark the common origin of two separate somatosensory pathways, corresponding to dorsal and ventral streams for somatic perception (Dijkerman \& de Haan, 2007).

There is an important distinction between tactile sensation and muscular proprioception, due to the different spatial organization of the 
original receptors. Tactile sensation arises from receptors in the skin, which forms a continuous sensory sheet covering the entire body and is considered a single sense organ. In contrast, proprioceptive organs including muscle spindles, tendon organs, and joint receptors are discretely and discontinuously located in separate muscles and joints. That is, these two afferent systems show different segmentation at the receptor level. At the level of the primary somatosensory cortex (SI) and the primary motor cortex (M1), neuroscientific studies show that, generally, somatosensory and motor representations follow anatomical divisions of the body, having receptive fields confined to single fingers or limbs. This gives rise to the metaphor of a "homunculus" (Penfield \& Boldrey, 1937; see also Blankenburg, Ruben, Meyer, Schwiemann, \& Villringer, 2003). However, the representations in SI and M1 have quite different organizing principles. Although both have comparable gross somatotopy, fine somatotopy differs sharply between the two areas. While the receptive field of each neuron of SI corresponds to a small well-defined part of the cutaneous surface (Blankenburg et al., 2003), M1 representations of the different body parts strongly overlap. The map in M1 is not as well segregated and segmented as that in SI. This difference may seem surprising, since it appears to be the inverse of the corresponding peripheral organization. That is, continuous skin in the periphery is segmented in the sensory cortex. In contrast, discrete muscles are represented in overlapping fashion in the motor cortex. Why, in particular, does the motor cortex not use the discrete, segmented representation of the motor periphery? According to one classical idea, skilled actions impose an additional functional organization because of the sets of body parts that work together in movements, and the motor cortex is organized in terms of action synergies, rather than muscles (Lemon, 1988). For example, when I move my forearm my hand and fingers follow. In contrast, if someone touches my forearm, this usually does not tell me anything about the sensation in the hand and fingers. Accordingly, M1 seems to be organized for representing muscle groups and patterns of movement rather than individual muscles (Lemon, 1988). The pattern of activation in M1 suggests that the control of any finger movement recruits a population of neurons distributed throughout M1, rather than a segregated population that would map point-to-point distinct movements based on a somatotopic organization (Schieber \& Hibbard, 1993). Muscles are grouped together to form functionally coherent units (Hlustik, Solodkin, Gullapalli, Noll, \& Small, 2001), producing considerable overlap in the cortical territories of adjacent body parts (Rathelot \& Strick, 2006).

Not only does the somatotopical organization vary between somatosensory and motor representations, but somatosensory processing itself varies depending on whether it is action related or recognition related (Dijkerman \& de Haan, 2007). The physiological organization of the somatosensory system reflects the eventual use of the somatosensory information. It can lead either to conscious perceptual judgements about any bodily feature or to actions that are programmed on the basis of information about the position and the size of the limbs. The former involves mainly the posterior insula and the posterior parietal cortex, whereas the latter involves the posterior parietal cortex. It remains unclear, however, whether these dual representations, which are clearly dissociable, nevertheless share a common principle for segmenting the body into parts.

Here we approach the problem of body mereology from the point of view of the primary sensorimotor mechanisms of the body. From this viewpoint, the segmentation of the body into parts may partly derive from the organization of the somatosensory system, from the organization of the motor systems, or from extrinsic perceptual factors such as visual discontinuities. On the one hand, the problem of segmenting the sensing body begins with the fact that the receptor surface (i.e., the skin) forms a continuous sheet. Here, relationships between the body parts are based on spatial contiguity in a cortical map. This representation seems not to support any concept of body part category. Instead, categories might arise at a higher level of integration or 
abstraction, perhaps cross-modally through viewing the physical discontinuities of one's own body structure.

On the other hand, the starting point for the mereology of the acting body is the set of muscles that I can voluntarily move. At first sight one might imagine that the acting body is intrinsically segmented, since muscles are not continuous or contiguous with each other, in the way the skin is. However, very few actions involve a single muscle alone: Muscles work in functional groups to achieve actions. That is, action structures the mental representation of the body into functional units, according to which body parts move together. Joints may be particularly important landmarks for motor mereology, since they allow relative movement of two body parts. From this perspective, we investigate whether somatosensory mereology and motor mereology indeed generate different relations and boundaries between body parts, how these two principles of organization interact, and what role vision plays.

We investigate segmentation effects in tactile and visual perception, comparing geometric perception tasks within and between body parts to give an implicit, quantitative measure of body segmentation. Body representation influences tactile perception in several ways. For instance, estimating the distance between two tactile stimuli can be biased by proprioceptive experience of those body parts (de Vignemont, Ehrsson, \& Haggard, 2005a). The representation of the body functions as a template for tactile perception (Martin, 1992). However, very few studies have investigated how body part segmentation influences tactile perception. How do boundaries structure the continuous tactile field of the skin? The arrangement of receptors in the skin varies considerably between different body parts (Weinstein, 1968). However, the density of receptors cannot explain why tactile localization is improved close to reference points (Weber, 1826/1978). Anatomical landmarks such as joints could play such a role and thus structure the continuous field (Bermudez, 1998; Flach \& Haggard, 2006). Cholewiak and Collins (2003) indeed found improved tactile localization close to joints, and also close to arbitrary anchor points artificially induced by presenting stimuli of frequency different from those used for tactile loci. Although joints have been the focus of research for tactile localization of a single point (Boring, 1942; Cholewiak, 1999; Cholewiak, Brill, \& Schwab, 2004; Cholewiak \& Collins, 2003; Parrish, 1897; Weber, 1826/1978), they have not been studied as possible determinants of distance perception between two points that span body segments. Green (1982) showed that perceived tactile distance varied with body site and orientation, but he did not consider tactile distances between two stimuli located in different body parts. If joints are indeed anatomical landmarks that play a role in segmenting the body into parts at the somatosensory level, one would expect tactile distances between two stimuli that span body parts across the joint to be perceived as further apart than tactile distances between two stimuli within a single body part. Here, we investigate the role of the wrist joint for both sensory and motor mereology.

We also investigate the possible sources of segmentation effects. Joints mark a visual discontinuity in the structure of the body, even though the skin covering them provides a continuous tactile field. Several studies show the importance of the interaction between vision and touch (Driver $\&$ Spence, 1998). In particular, tactile distance perception is influenced by purely visual experience of the stimulated body parts (Taylor-Clarke, Jacobsen, \& Haggard, 2004). Thus, segmentation effects in tactile perception could arise cross-modally, from visual segmentation of body structure. We therefore also investigate whether segmentation effects occur in purely visual perception of the body or structurally similar stimuli. If purely visual segmentation effects are found, they could potentially explain tactile segmentation via cross-modal links.

\section{EXPERIMENT 1}

Experiment 1 compared the judgement of tactile distances on the palm or forearm, with distances 
spanning these two body parts across the wrist joint. If tactile perception is organized in terms of body parts, one would expect a significant difference between the distance judgements of cross-joint and within-body part distances. It would show that the wrist constitutes a natural boundary between palm and forearm, giving rise to the tactile sensation that the stimuli on the two body parts feel further apart. Since the underlying receptor sheet of the skin is continuous, such segmentation effects would represent a contextual modulation of tactile perception by a cognitive representation of one's own body.

\section{Method}

\section{Participants}

A total of 8 healthy volunteers participated in the experiment ( 5 females; $20.6 \pm 0.9$ years, mean \pm standard deviation). Participants were paid $£ 4$, and all gave informed consent.

\section{Materials, design, and procedure}

Two simultaneous tactile stimuli were delivered by a caliper to the ventral forearm, to the palm, or to both forearm and palm across the wrist joint. The centre of these three tactile distances was situated $50 \mathrm{~mm}$ proximal to the wrist for stimulation of the ventral forearm, $50 \mathrm{~mm}$ distal to the wrist for stimulation of the palm, and over the wrist joint itself for cross-joint distances. The actual distance between points was 40,50 , or $60 \mathrm{~mm}$, and each distance was presented five times in a fixed random order. The blindfolded participant sat at a table, with the right forearm and the right hand lying comfortably extended in the frontoparallel plane. Participants gave verbal estimates of the perceived distance in millimetres.

\section{Results and discussion}

Performance was measured as judgement error (perceived distance - stimulus distance). We compared the mean judgement error for distances on the palm alone, on the forearm alone, or across the wrist. Note that each participant may use a different internal distance standard, or "subjective $\mathrm{mm}$ ", but this will not affect comparisons between body parts in a repeated measures design. A 3 (body part: palm, forearm, across the wrist) by 3 (distance: 40, 50, $60 \mathrm{~mm}$ ) repeated measures analysis of variance (ANOVA) revealed a general underestimation of distance, also called "spatial compression" (Green, 1982): ANOVA intercept, $F(1,7)=7.23, p<.03, \eta_{\mathrm{p}}^{2}=.64(M=-8.25$, $S E=3.07)$.

There was a main effect of body part, $F(2,14)$ $=11.75, p<.001, \eta_{\mathrm{p}}^{2}=.63$. Judgement error was greater for the forearm than the palm, $t(7)=2.81$, $p<.03$, consistent with the lower acuity of the arm compared to the palm (Green, 1982; Weber, 1826/1978). Crucially, however, judgement error was greater within body parts than it was across parts in the comparison both of the forearm distance with the distance across the wrist, $t(7)=$ 4.05, $p<.005$, and of the palm distance with the distance across the wrist, $t(7)=2.71, p<$ .03. This finding is not explicable in terms of acuity differences (see Figure 1). There was also a main effect of distance, $F(2,14)=4.23, p<$ $.04, \eta_{\mathrm{p}}^{2}=.37$, and an interaction of body part by distance, $F(4,28)=4.66, p<.005, \eta_{\mathrm{p}}^{2}=.40$. Increasing distances gave rise to larger judgement

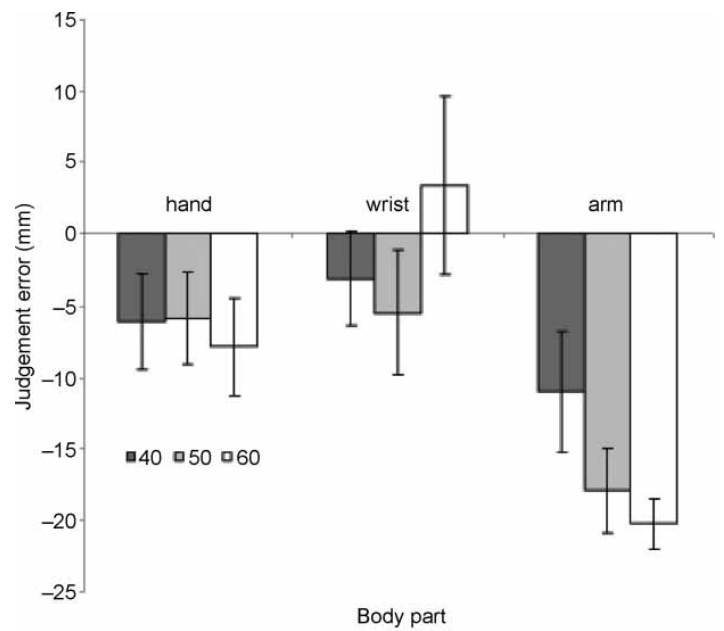

Figure 1. Mean judgement error for tactile distance between two points presented on the hand, on the arm, or across the wrist, for the three distances (bars indicate standard errors). 
errors on the forearm than on the palm or across the wrist.

Participants overestimated the distance between two points when they spanned two body parts, relative to equivalent distances within a single body part (the palm or the forearm). A simple model of the tactile field as a continuous receptor surface might predict that distances across the joint would produce a perceived distance intermediate between that on the two adjoining body parts. In contrast, our results show a superadditive interaction, suggesting that the wrist joint itself serves as a structuring element that alters perception of the underlying tactile field of the skin. Distances across the wrist joint are relatively overestimated, implying a perceptual repulsion of the two tactile stimuli away from the wrist joint. Studies of tactile localization show better localization around joints than in other regions, even when sets of stimuli span the joints (Cholewiak \& Collins, 2003). Joints serve as attractors for localization (Flach \& Haggard, 2006). Interestingly, they have an opposite, repulsive effect on tactile distance judgement.

\section{EXPERIMENT 2}

Experiment 1 highlighted variations in tactile distance judgements across the wrist. However, joints are the basis of movement, as well as being morphological landmarks. Thus, the structuring effect of joints on tactile distance perception could reflect either perceptual salience or motor salience. To disentangle these two aspects, we investigated whether voluntary movements of the wrist joint modulate tactile distance judgements.

\section{Method}

\section{Participants}

A total of 16 undergraduates from the Institute of Cognitive Neuroscience, University College London participated in the experiment (10 females; $28.2 \pm 4.7$ years), none of whom had participated in the previous study. They were paid $£ 4$ and participated on the basis of informed consent.

\section{Materials, design, and procedure}

Participants received tactile distance stimulation with 8 miniature solenoids with a conical tip of $1 \mathrm{~mm}^{2}$. They were grouped in two arrays of 4. Within each array, the centres of adjacent solenoids were $15 \mathrm{~mm}$ apart, and the gap across the wrist between the ends of the arrays was $45 \mathrm{~mm}$. Distances tested were 15, 30, and $45 \mathrm{~mm}$ within a single body part (palm or forearm) and 45, 75, and $105 \mathrm{~mm}$ across the wrist. The $45-\mathrm{mm}$ distance thus had a special interest for comparing perception of an identical distance within or between body parts and formed the focus of our analysis. Distances and locations of the stimuli were presented in random order, so that the participants were not aware of the special significance of the 45-mm stimuli.

There were two conditions: an action condition and a rest control condition. In the action condition, participants made wrist flexion-extension movements to imitate a model arm shown on a computer screen. The model arm made step-like wrist movements to $120^{\circ}, 150^{\circ}, 180^{\circ}, 210^{\circ}$, and $240^{\circ}$ wrist angles in random order, at intervals of $1 \mathrm{~s}$. The experimenter verified that the participant performed accurate and rapid movements to follow the model. Tactile stimulation was always delivered when the wrist angle was $180^{\circ}$ (the hand aligned with the arm). In the control condition, the participant's arm remained continually in the $180^{\circ}$ position. Each participant received each stimulus distance eight times in random order in each condition.

Participants sat at a table, with the right forearm comfortably extended with the palm vertical. An occluding screen prevented direct viewing of forearm and hand. Participants were required to match each stimulation distance with visually displayed lines. Participants viewed a set of 52 lines varying in length from $10 \mathrm{~mm}$ to $112 \mathrm{~mm}$, each coded by a letter and a colour. The lines were presented at approximately the same distance from the eye as from the wrist. Displays were randomly presented in ascending 
or descending order of length to prevent response code learning. Participants made verbal responses to indicate which line best matched the tactile distance.

\section{Results and discussion}

Performance was measured in terms of judgement error (perceived distance - stimulus distance). A 3 (body part: palm, forearm, across the wrist) by 2 (movement: rest, action) repeated measures ANOVA was conducted on the $45 \mathrm{~mm}$ distance stimulus, which was common to all the body sites. As in the previous experiment, there was a general underestimation of distance, ANOVA intercept, $F(1,15)=21.07, p<.0001, \eta_{\mathrm{p}}^{2}=.58$ $(M=-8.99, S E=1.96)$. There was a main effect of body part, $F(2,14)=16.63, p<.0001$, $\eta_{\mathrm{p}}^{2}=.70$. Judgement error was greater for the forearm than the palm, $t(15)=3.03, p<.008$. Moreover, judgement error was greater within body part than it was across parts in the comparison both of the forearm with the wrist, $t(15)=$ $5.25, p<.0001$, and of the palm with the wrist, $t(15)=3.49, p<.003$. This effect replicated the finding of Experiment 1, despite differences in the overall estimates between the two experiments. These may be explained by differences in response mode and stimulus delivery. Distances across joints were overestimated relative to equivalent distances within a single body part.

There was no significant effect of action, $F(1$, $15)=0.64, p=.44, \eta_{\mathrm{p}}^{2}=.04$, but there was an interaction of body part with action, $F(2,14)=$ $5.08, p<.02, \eta_{\mathrm{p}}^{2}=.42$. Action reduced the tendency to relatively overestimate distances across the wrist, as shown in Figure 2.

Experiment 2 demonstrates that action influences the segmentation of the body surface. The wrist was in the same position in both action and rest trials, and the motor task involved equal flexion and extension phases, with an average wrist angle equal to the rest control condition. Therefore, the interaction between action and body part must reflect an effect of action on the spatial representation of tactile stimuli, rather than a simple change in actual stimulus location

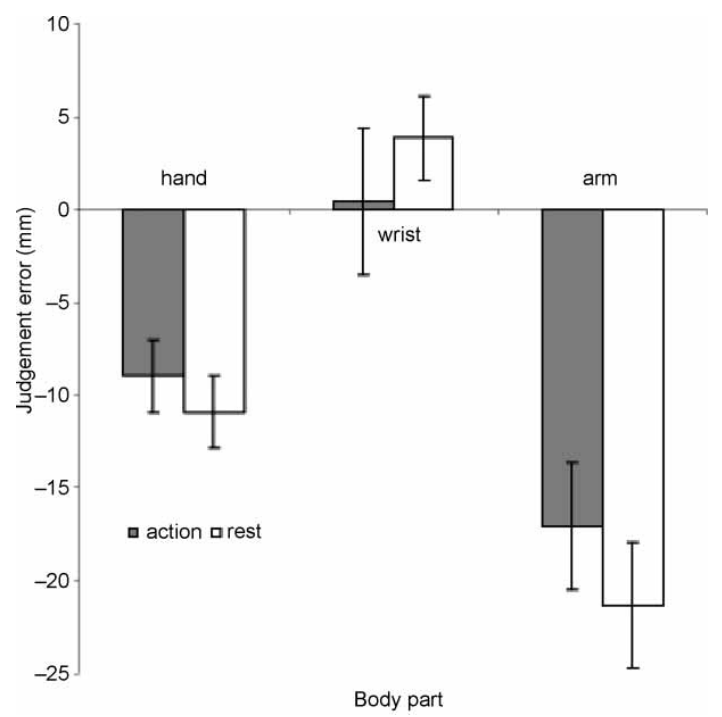

Figure 2. Mean judgement error for tactile distance between two points presented on the hand, on the arm, or across the wrist, in the action condition and while static (bars indicate standard errors).

in external space. The reducing effect of action on distances across the wrist suggests that the segmentation of the body for action is less dramatic than that for perception. While perceptual representation appears to involve segmentation, action representations may involve unification between body parts.

\section{EXPERIMENT 3}

The previous two experiments show segmentation effects in tactile perception. Are these effects specific to bodily sensations or could they result from a purely visual segmentation of the body, transferring to touch via a novel cross-modal link? To assess this possibility, we conducted two further experiments. In Experiment 3, participants were asked to make judgements of distance between solely visually presented stimuli on their own arm. As in Experiment 1, we compared the judgement of distances on the palm or forearm, with distances spanning these two body parts across the wrist joint. If the segmentation effect in tactile perception arises from visual segmentation 
of the body, one would expect to find the same effect in visual perception.

\section{Method}

\section{Participants}

A total of 8 undergraduates from the Radboud University, Nijmegen, participated in the experiment ( 6 females; $23.6 \pm 2.3$ years). They were paid $€ 4$ and participated on the basis of informed consent.

\section{Materials, design, and procedure}

Two points of light were projected onto the surface of the participant's body. The stimulus was presented by a projector connected to a computer, which controlled the presentation of the lights. In order to determine the exact location to present the images, a calibrator was utilized. The calibrator was a $19-\mathrm{cm}$ ruler, which had two light sensors at the ends. Participants rested their arm on a flat metal ramp at a $5^{\circ}$ incline, which ensured that participants were able to keep their hands completely open for the duration of the experiment.

Stimuli were presented across the wrist, only on the palm, or only on the forearm. The stimulus points were two white dots, $1 \mathrm{~mm}$ in diameter, and were presented for $1 \mathrm{~s}$. Distances between points were 40,50 , or $60 \mathrm{~mm}$. The points were presented $50 \mathrm{~mm}$ proximal to the wrist for presentation on the forearm, $50 \mathrm{~mm}$ distal to the wrist for presentation on the palm, and over the wrist joint itself for cross-joint distances. Each distance was presented five times, in a different random order for each participant. On each trial, participants gave a verbal estimate of the distance in millimetres. The experimenter noted the answer and initiated the next trial until completion.

\section{Results and discussion}

A 3 (body part: palm, forearm, across the wrist) by 3 (distance: 40, 50, $60 \mathrm{~mm}$ ) repeated measures ANOVA was conducted on judgement error (perceived distance - stimulus distance). Unlike the tactile estimation experiments, but consistent with the existing literature (Gescheider, 1997; Stevens, 1975), there was no general underestimation of distance: ANOVA intercept, $F(1,7)=$ 0.97, $p=.36, \eta_{\mathrm{p}}^{2}=.12(M=-1.74, S E=$ 1.76). There was no significant effect of body part, $F(2,14)=0.56, p=.58, \eta_{\mathrm{p}}^{2}=.07$, nor of distance, $F(2,14)=3.31, p=.07, \eta_{\mathrm{p}}^{2}=.32$. There was, however, an interaction of body part by distance, $F(4,28)=2.72, p<.05, \eta_{\mathrm{p}}^{2}=.28$. This was driven by judgement errors for the arm, which were more accurate with increasing distances (see Figure 3). There was a slight difference in judgement errors for $40 \mathrm{~mm}$ and $50 \mathrm{~mm}$ distances, $t(7)=2.00, p<.09$, and a significant difference between $40 \mathrm{~mm}$ and $60 \mathrm{~mm}$ distances, $t(7)=3.08, p<.02$. No other differences were significant (all $t \mathrm{~s}<1.0$ ).

In contrast with Experiment 1, there was no segmentation effect on judgements of visual distance between two points. It did not make a difference whether the visual stimuli were presented within a single body part or on two body parts across the wrist. Tactile perception of distance thus does not follow the same principles as visual perception.

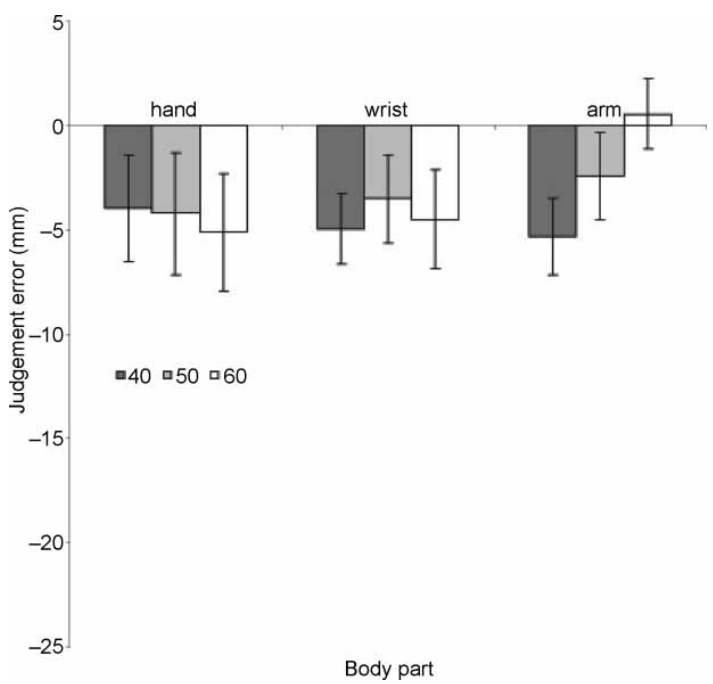

Figure 3. Mean judgement error for visual distance between two points presented on the hand, on the arm, or across the wrist, for the three distances (bars indicate standard errors). 


\section{EXPERIMENT 4}

As in Experiment 3, we were interested in the specificity of the segmentation effects, this time investigating the influence of the type of object (body vs. nonbody), instead of the modality (touch vs. vision). Tactile stimulations can be applied solely on the body so we cannot compare directly with stimulation on a nonbody object. Nonetheless, we investigated the segmentation effect on visual stimulation of a nonbody stimulus. The study also presents an opportunity to investigate whether structural parts influence visual distance estimation. In this experiment, participants were asked to make judgements of distance based on visually presented stimuli to a neutral shape.

\section{Method}

\section{Participants}

A further 8 undergraduates from the Radboud University, Nijmegen, participated in the experiment (5 females; $22.1 \pm 2.2$ years); none had participated in Experiment 3. They were paid $€ 4$ and participated on the basis of informed consent.

\section{Materials, design, and procedure}

All details were as described in Experiment 3, only the images were not projected onto the participants' body, but onto a fixed neutral shape. The shape was chosen so as to have some similarity to the body - a funnel, resembling the hand-shape, was placed on top of a long rectangle. The "joint" was indicated by a line segmenting the funnel from the rectangle (see Figure 4).

\section{Results and discussion}

A 3 (object part: funnel, rectangle, span) by 3 (distance: 40, 50, $60 \mathrm{~mm}$ ) repeated measures ANOVA was conducted on judgement error (perceived distance - stimulus distance). There was a general underestimation of distance: ANOVA intercept, $F(1,7)=22.28, p<.002, \eta_{\mathrm{p}}^{2}=.76(M=-9.04$, $S E=2.11)$. There was no significant effect of object part, $F(2,14)=2.44, p=.12, \eta_{\mathrm{p}}^{2}=.26$.

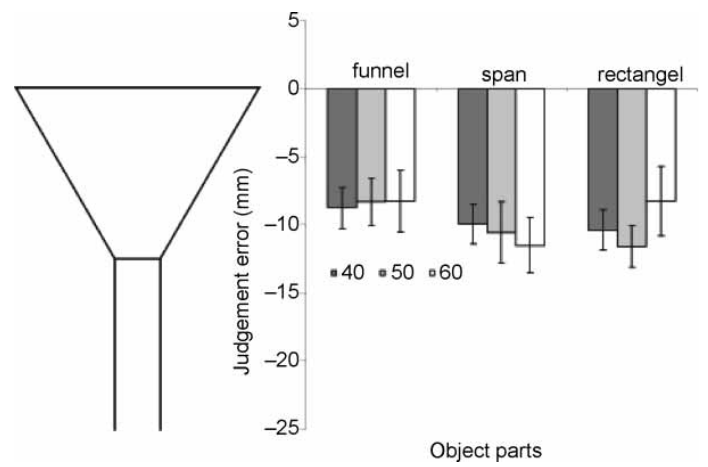

Figure 4. Mean judgement error for visual distance between two points presented on an object-on a funnel, on a rectangle, or spanning parts-for the three distances (bars indicate standard errors).

There was also no significant main effect of distance, $F(2,14)=0.08, p=.92, \eta_{\mathrm{p}}^{2}=.01$, and no significant part by distance interaction, $F(4,28)=1.99, p=.12$, $\eta_{\mathrm{p}}^{2}=.22$ (see Figure 3). In particular, there was no evidence of any segmentation effects on visual distance judgements

\section{GENERAL DISCUSSION}

On the one hand, the body surface may be viewed as an undifferentiated cutaneous sheet, without categorical divisions corresponding to anatomical body parts. On the other hand, we naturally think and speak of bodies in terms of their parts (Majid et al., 2006). Our study showed that body segmentation arises as early in processing as the levels of integrating two tactile sensations in a geometric judgement. Geometric perceptions of stimuli applied to the skin are strongly influenced by the segmentation of the body. First, we showed that the tactile surface is not homogeneous. Joints play the role of body landmarks that segment the body into parts and increase judgements of tactile distance. Second, these segmentation effects are weakened by action. In one sense, action brings body parts together into coherent functional units. Third, these segmentation effects are specific to tactile sensations. Control experiments with visual stimuli showed that 
visual distance judgements are not influenced by the joint, or by any visual boundary. Therefore, tactile segmentation seems to be intrinsic to touch and cannot arise indirectly as a result of cross-modal links from vision.

What delineates body part categories? We showed the importance of the wrist in segmenting the upper limb into hand and arm by using tactile distance judgements (Green, 1982; Weber, 1826/ 1978). This task involves perception of tactile pattern and geometry. These percepts seem to involve factors beyond the primary somatosensory map. As noted by Weber (1826/1978), judgements of spatially extended patterns do not depend only on the physical extent, but also on other factors, such as the body part and the orientation of the pattern on the surface of the body. Cholewiak (1999) also showed that it was related to the temporal delay between the two tactile stimulations. We suggest a further factornamely, the segmentation of the body surface into categorical body parts. Tactile perception depends on the representation of the volumetric articulated body, in addition to the skin surface itself. Specifically, perceived tactile distance increases when the tactile stimuli are on distinct body parts in comparison to when they are on a single body part. This result is reminiscent of the "category boundary effect" found in studies of categorization: heightened discriminability between categories and reduced discriminability within categories (Pastore, 1987). In the same way that two shades of colour seem more different if they belong to two distinct colour categories than if they do not, two tactile stimuli feel more separated if they are applied to two distinct body part categories. Kosslyn et al. (1989) made a distinction in visual perception and imagery, which is of particular interest here. They distinguished between "coordinate" spatial representations, based on metric location information, and "categorical" spatial representations, based on more abstract spatial relations between objects or parts of objects. Our results show that the metric representation of the body is influenced by a categorical body representation. This categorical body representation may be processed in the left hemisphere, which is specialized in categorical spatial relations (Kosslyn et al., 1989). Lesions here may result in deficits of structural body representation such as autotopagnosia (Buxbaum \& Coslett, 2001). Therefore, the neural representation of touch is not merely organized according to principle of spatial contiguity and continuity. It is already structured into categorical body parts at the level where tactile stimuli are integrated and their spatial relations represented.

The second experiment replicated this segmentation effect in tactile distance judgements with a new group of participants, using an alternative form of judgement. It also showed that body segmentation is influenced by action. Action appeared to homogenize the body surface, by reducing the relative distortion of tactile distances across the wrist joint. This result suggests that the relative overestimation of cross-joint distances cannot itself be a motor effect. If that were the case, the relative overestimation of cross-joint distances would have been increased by action whilst we found the opposite effect. Therefore, we suggest that the relative overestimation of cross-joint distance judgements arises from a perceptual categorization of the body into parts. In contrast, when the tactile information is processed during a motor task (Experiment 2, action condition), we found that the structuring role of the wrist joint was reduced, and the relative overestimation of cross-joint distances was attenuated. That is, action reduced the tendency to segment the body into categorical parts and to overestimate the distance between them.

Representation of the body for action therefore reduces the bias inherent to the categorical distinction of body parts. Action requires a unified representation of the body that puts body parts back together. Motor synergies between several effectors require an integrative, rather than a differentiated, representation of the relevant body parts. A similar conclusion regarding the integrative effects of action on body representation was reached by Tsakiris, Prabhu, and Haggard (2006) in a study of the rubber hand illusion. The authors showed that the illusory ownership of a rubber hand generated by synchronous visual 
stimulation of the rubber hand and stimulation of the participant's hand was local, and confined to the stimulated finger, when based on afferent information. When the same finger was actively moved, however, the illusion spread to other fingers. Action appeared to induce a more global, integrated body representation, while afferent information resulted in representation of effectively unconnected individual body parts. The difference between the two conditions, rest and action, is consistent with the model of the dual somatosensory pathways proposed recently by Dijkerman and de Haan (2007). Our result suggests that segmentation into parts is a property of the ventral-perceptual body representation pathway, rather than the dorsal-action body representation pathway.

What is the source of the segmentation effect? First, we excluded two hypotheses, which attribute tactile segmentation to other, nontactile factors. On one view, tactile segmentation results from the possibility for two body segments to move separately, demonstrating that they are dissociable. According to a second hypothesis, it results from the visual discontinuity between two body segments. However, in Experiment 2, we showed that action brings body parts closer, instead of pulling them further apart. As for the second hypothesis, we did not find any segmentation effect for the visual percept of the body in Experiment 3. There was no significant difference between visual distances within and across body parts. Estimates of the spatial distance between the two points were not affected by the surrounding visual display of the participant's hand and forearm. Although we did not expect this result, we would like to provide a possible interpretation for this lack of visual segmentation effect.

We would like to suggest that touch and vision differ in how they process spatial properties. The tactile perception of spatial relationships is always mediated by the representation of the body, whereas visual experiences derive their spatial properties from the visual field itself without further modulation. Tactile sensations are always bodily sensations. There is an intrinsic link between touch and the body that cannot be found for vision. Indeed, vision can be about the body, but also about any other object. Consequently, it is possible to compute the distance between the two white dots in Experiment 3 independently of the fact that they are visually displayed on the participant's arm and hand. Experiment 4 indeed showed the same lack of segmentation effect for the visual percept of a neutral shape. In Experiment 3, participants could effectively ignore the visual display of the body on which the stimuli were presented and could perform the visual task in a body-independent space as in Experiment 4. The bodily background cannot be removed from touch (Experiments 1 and 2) in the way it can from vision.

However, the nature of the link between body representation, proprioception, and touch remains controversial. Some philosophers have argued that the body functions as a template for tactile perception-that is, tactile perception of the spatial properties of an object relies on the proprioceptive experience of similar properties. This is particularly salient for shape in active touch: We feel the circularity of a glass because we feel the circularity of the motion of our hand (Martin, 1992). The relation between touch and the body can then be explained in terms of a fusion of tactile and proprioceptive representations. However, veridical tactile perception does not necessarily match proprioceptive sensations, and in our experiments only passive touch was involved. Instead, we would like to suggest that the tactile field does indeed reflect the body, but does so because of the structuring effect of a cognitive body representation. Tactile stimuli are not judged relatively to each other, but rather in a bodily frame of reference (Cholewiak, 1999; Green, 1982). However, the spatial organization is intrinsically tactile and does not arise from proprioception.

In summary, we have provided preliminary experimental evidence to support a previous theoretical distinction between two kinds of body mereology (de Vignemont et al., 2005b). The somatosensory mereology relies on a structured description of the body, categorically organized. The joints play the role of body landmarks. In distance judgement, the joint delineates body parts, leading to a "category 
boundary effect". In contrast, motor mereology relies on a more unified and consistent representation of the body. Action reduces the "boundary effect". We suggest that action produces a distributed and integrated experience of the body, linking together the body parts involved in motor synergies. Consequently, the mereology of the sensing body and the mereology of the acting body appear to have the functions of differentiating and grouping body parts, respectively. Finally, more work is required to generalize our result beyond the current study. We focused here on the wrist joint for a good reason. Since it can move in both flexion and extension, its actions need not alter the average distance in external space between two points that span it. This allowed us to assess effects of action on tactile distance without introducing external spatial confounds, or possible biases. However, it would be valuable to study other joints in the body to assess generality.

Original manuscript received 28 February 2007 Accepted revision received 7 February 2008 First published online 6 May 2008

\section{REFERENCES}

Andersen, E. S. (1978). Lexical universals of body-part terminology. In J. H. Greenberg (Ed.), Universals of buman language (pp. 335-368). Stanford, CA: Stanford University Press.

Bermudez, J. L. (1998). The paradox of self-consciousness. Cambridge, MA: MIT Press.

Biederman, I. (1987). Recognition-by-components: A theory of human image understanding. Psychological Review, 94, 115-147.

Blankenburg, F., Ruben, J., Meyer, R., Schwiemann, J., \& Villringer, A. (2003). Evidence for a rostral-tocaudal somatotopic organization in human primary somatosensory cortex with mirror-reversal in areas 3b and 1. Cerebral Cortex, 13, 987-993.

Boring, E. G. (1942). Sensation and perception in the history of experimental psychology. New York: Appleton-Century.

Brown, C. H. (1976). General principles of human anatomical partonomy and speculations on the growth of partonomic nomenclature. American Ethnologist, 3, 400-424.
Brown, C. H. (2005). Hand and arm. In M. Haspelmath, M. Dryer, D. Gil, \& B. Comrie (Eds.), The world atlas of language structures (pp. 522-525). Oxford, UK: Oxford University Press.

Buxbaum, L. J., \& Coslett, H. B. (2001). Specialized structural descriptions for human body parts: Evidence from autotopagnosia. Cognitive Neuropsychology, 18, 289-306.

Buxbaum, L. J., Giovannetti, T., \& Libon, D. (2000). The role of the dynamic body schema in praxis: Evidence from primary progressive apraxia. Brain and Cognition, 44, 166-191.

Cholewiak, R. W. (1999). The perception of tactile distance: Influences of body site, space, and time. Perception, 28, 851-875.

Cholewiak, R. W., Brill, J. C., \& Schwab, A. (2004). Vibrotactile localization on the abdomen: Effects of place and space. Perception E Psychophysics, 66, 970-987.

Cholewiak, R. W., \& Collins, A. A. (2003). Vibrotactile localization on the arm: Effects of place, space, and age. Perception E Psychophysics, 65, 1058-1077.

de Vignemont, F., Ehrsson, H., \& Haggard, P. (2005a). Bodily illusions modulate tactile perception. Current Biology, 15, 1286-1290.

de Vignemont, F., Tsakiris, M., \& Haggard, P. (2005b). Body mereology. In G. Knoblich, I. M. Thornton, M. Grosjean, \& M. Shiffrar (Eds.), Human body perception from the inside out (pp. 147-170). Oxford, UK: Oxford University Press.

Dijkerman, H. C., \& de Haan, E. H. F. (2007). Somatosensory processes subserving perception and action. Behavioral and Brain Sciences, 30, 189-201.

Driver, J., \& Spence, C. (1998). Attention and the cross-modal construction of space. Trends in Cognitive Sciences, 2, 254-262.

Flach, R., \& Haggard, P. (2006). The cutaneous rabbit revisited. Journal of Experimental Psychology: Human Perception and Performance, 32, 717-732.

Gallagher, S., \& Cole, J. (1995). Body image and body schema in a deafferented subject. Journal of Mind and Behavior, 16, 369-390.

Gescheider, G. A. (1997). Psychophysics: The fundamentals (3rd ed.). Hillsdale, NJ: Lawrence Erlbaum Associates.

Goldenberg, G. (1995). Imitating gestures and manipulating a manikin. The representation of the human body in ideomotor apraxia. Neuropsychologia, 33, 63-72.

Green, B. G. (1982). The perception of distance and location for dual tactile pressures. Perception $\mathcal{E}^{\circ}$ Psychophysics, 31, 315-323. 
Hlustik, P., Solodkin, A., Gullapalli, R. P., Noll, D. C., \& Small, S. L. (2001). Somatotopy in human primary motor and somatosensory hand representations revisited. Cerebral Cortex, 11, 312-321.

Kosslyn, S. M., Koenig, O., Barrett, A., Cave, C. B., Tang, J., \& Gabrieli, J. D. (1989). Evidence for two types of spatial representations: Hemispheric specialization for categorical and coordinate relations. Journal of Experimental Psychology: Human Perception and Performance, 15, 723-735.

Laiacona, M., Allamano, N., Lorenzi, L., \& Capitani, E. (2006). A case of impaired naming and knowledge of body parts. Are limbs a separate sub-category? Neurocase, 12, 307-316.

Lemon, R. N. (1988). The output map of the primate motor cortex. Trends in Neuroscience, 11, 501-506.

Levinson, S. C. (2006). Parts of the body in Yeli Dnye, the Papuan language of Rossel Island. Language Sciences, 28, 221-240.

Majid, A., Enfield, N. J., \& van Staden, M. (Eds.). (2006). Parts of the body: Cross-linguistic categorisation [Special issue]. Language Sciences, 28(2-3).

Martin, M. (1992). Sight and touch. In T. Crane (Ed.), The content of experience (pp. 199-201). Cambridge, UK: Cambridge University Press.

Milner, D., \& Goodale, M. A. (1995). The visual brain in action. New York: Oxford University Press.

Morrison, J. B., \& Tversky, B. (2005). Bodies and their parts. Memory and Cognition, 33, 696-709.

Paillard, J. (1999). Body schema and body image-a double dissociation in deafferented patients. In G. N. Gantchev, S. Mori, \& J. Massion (Eds.), Motor control, today and tomorrow (pp. 197-214). Sophia, Bulgaria: Academic Publishing House.

Parrish, C. S. (1897). Localization of cutaneous impressions by arm movement without pressure upon the skin. American Journal of Psychology, 8, 250-267.

Pastore, R. E. (1987). Categorical perception: Some psychophysical models. In S. Harnad (Ed.), Categorical perception: The groundwork of cognition (pp. 29-52). New York: Cambridge University Press.

Penfield, W., \& Boldrey, E. (1937). Somatic motor and sensory representation in the cerebral cortex of man as studied by electrical stimulation. Brain, 60, 339-448.

Rathelot, J. A., \& Strick, P. L. (2006). Muscle representation in the macaque motor cortex: An anatomical perspective. Proceedings of the National Academy of Sciences, 103, 8257-8262.

Reed, C. L., McGoldrick, J. E., Shackelford, R., \& Fidopiastis, C. (2004). Are human bodies represented differently from other animate and inanimate objects? Visual Cognition, 11, 523-550.

Rossetti, Y., Rode, G., \& Boisson, D. (1995). Implicit processing of somaesthetic information: A dissociation between where and how? Neuroreport, 6, 506-510.

Schieber, M. H., \& Hibbard, L. S. (1993). How somatotopic is the motor cortex hand area? Science, 261, 489-492.

Schwoebel, J., \& Coslett, H. B. (2005). Evidence for multiple, distinct representations of the human body. Journal of Cognitive Neuroscience, 17, 543-553.

Sirigu, A., Grafman, J., Bressler, K., \& Sunderland, T. (1991). Multiple representations contribute to body knowledge processing. Evidence from a case of autotopagnosia. Brain, 114, 629-642.

Stevens, S. S. (1975). Psychophysics. New York: Wiley \& Sons.

Suzuki, K., Yamadori, A., \& Fujii, T. (1997). Categoryspecific comprehension deficit restricted to body parts. Neurocase, 3, 193-200.

Taylor-Clarke, M., Jacobsen, P., \& Haggard, P. (2004). Keeping the world a constant size: Object constancy in human touch. Nature Neuroscience, 7, 219-220.

Tsakiris, M., Prabhu, G., \& Haggard, P. (2006). Having a body versus moving your body: How agency structures body-ownership. Conscious and Cognition, 15, 423-432.

Weber, E. H. (1978). The sense of touch (De Tactu, H. E. Ross, Trans.; Der Tastsinn, D. J. Murray, Trans.). New York: Academic Press. (Original work published 1826.)

Weinstein, S. (1968). Intensive and extensive aspects of tactile sensitivity as a function of body part, sex and laterality. In D. R. Kenshalo (Ed.), The skin senses (pp. 195-218). Springfield, IL: Thomas. 